Check for updates

Cite this: RSC Adv., 2017, 7, 23735

Received 23rd March 2017

Accepted 23rd April 2017

DOI: $10.1039 / \mathrm{c} 7 \mathrm{ra03384b}$

rsc.li/rsc-advances

\title{
Isolation of functional mitochondria by inertial microfluidics - a new method to sort intracellular organelles from a small scale biological sample
}

\author{
C. Tesauro, (D) a B. Ferrando, (D) ${ }^{a}$ X. Ma, ${ }^{\text {ab }}$ M. L. Jepsen, ${ }^{\text {ab }}$ A. K. R. Ivarsen, ${ }^{\text {bc }}$ R. Frøhlich, ${ }^{a}$ \\ T. Stevnsner, ${ }^{a}$ B. R. Knudsen ${ }^{\text {ab }}$ and Y. P. Ho (iD *d
}

\begin{abstract}
Isolation of subcellular fractions from a limited amount of clinical sample is imperative for the continuous advancement of biological and clinical research. For example, analyses of pure and functional mitochondria from patient samples are expected not only to expand our knowledge towards the basic biological mechanisms of mitochondrial function, but also to elucidate how mitochondria are involved in the development of diseases such as cancers, premature aging syndromes, diabetes and neurodegenerative disorders. While currently available methods are mostly laborious and not suitable for small-scale analyses, we present a novel and facile approach to isolate mitochondria from limited amounts of biological samples by centrifugal-based differential migration. Furthermore, sorting cellular organelles by their intrinsic inertia enjoys the benefits of easy operation, undemanding equipment needs and continuous batch processing. Herein, we have successfully isolated functional mitochondria from crude cell lysate of less than 100 cells, which demonstrates the possibilities of promoting this methodology for detailed analyses of subcellular organelles, particularly when small-scale clinically relevant samples are considered.
\end{abstract}

\section{Introduction}

Isolation of subcellular fractions of low abundance has enabled more efficient identification and study of intracellular organelles of interest and is, consequently, essential for the continuous advancement of biology. For instance, isolation of mitochondria, the so-called "power houses" of the cells, may help to investigate the anabolic and catabolic processes in which the mitochondria are involved..$^{1-3}$ Elucidating the functions of mitochondria in detail is particularly important since defective mitochondria have been linked to several important

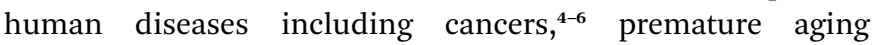
syndromes, ${ }^{7}$ diabetes $^{\mathbf{8 , 9}}$ and neurodegenerative disorders. ${ }^{\mathbf{1 0 , 1 1}}$ To this end, it becomes imperative to develop a platform capable of isolating functional mitochondria particularly from a small amount of sample, since in practice clinical samples are often limited in size. Indeed, analyses of mitochondria from healthy or sick tissue samples are expected not only to expand our

${ }^{a}$ Aarhus University, Department of Molecular Biology and Genetics, Aarhus, 8000, Denmark

${ }^{b}$ Aarhus University, Interdisciplinary Nanoscience Center (iNANO), Aarhus, 8000, Denmark

${ }^{c}$ Aarhus University, Department of Engineering, Aarhus, 8000, Denmark

${ }^{d}$ The Chinese University of Hong Kong, Division of Biomedical Engineering, Department of Electronic Engineering, Faculty of Engineering, Shatin, N.T., Hong Kong SAR, China.E-mail: ypho@ee.cuhk.edu.hk knowledge towards the basic biological mechanisms of mitochondrial function, but also to elucidate the role of mitochondria in human disease development. Currently available methods for isolation of intact mitochondria are mostly laborious, typically requiring cell lysis, fractionation, and purification of mitochondria from cytosol $^{12,13}$ and is therefore not suitable for small-sample analysis. For example, the densitygradient centrifugation is a widely used and robust approach, but often requires many labour-intensive steps, rendering it only practical for large-scale needs. The process is also complicated by the request of differential centrifugations and buffer exchange steps. Immunoisolation is highly specific, but it also demands multiple steps. Both methods are time- and sample-consuming, with steps performed in series resulting in significant sample loss during handling, which poses a remarkable barrier for the analysis of precious clinical samples.

Microfluidics, typically referred to as the science that deals with the behaviour and manipulation of fluids in the scale of tens to hundreds of micrometers, is able to minimize sample and reagent consumption, to shorten the reaction time with large surface-to-volume ratio, to reduce the cost, to enable portability, and to offer automation for minimal human intervention. ${ }^{\mathbf{1 4 - 1 7}}$ Therefore, this technology has enabled many new promising possibilities for biomedical and chemical applications. ${ }^{18}$ Owing to the advantages offered by microfluidics, as compared to conventional macro-scale technologies (e.g. 
centrifugation, flow cytometry, etc.), many microfluidics-based innovations have been proposed for rapid isolation of organelles from small amounts of samples. For instance, the microfluidic field flow fractionation device is developed for continuous separation of subcellular organelles by isoelectric focusing (IEF). ${ }^{19}$ However, the enrichment of organelles using IEF requires prior knowledge of the isoelectric point (pI) of the target organelles. Take mitochondria for example, previous reports have identified the pIs of mitochondria, but the obtained value varies depending on the source and method used for the characterization, ${ }^{20-23}$ making the isolation unreliable solely based on the pI. To address this issue, capillary IEF (CIEF) plus laser induced fluorescence ${ }^{24}$ presents an accurate determination of the pI of individual mitochondria by the use of internal standards. However, the specificity of mitochondrial sorting by cIEF relies heavily on the optimization of the applied electric field strength and demands an extended run time and a high power input. The long exposure to the electric field is likely to affect the organelles adversely and causes Joule heating. Furthermore, the equipment request is not well-suited for the standard biology or biomedical laboratory setup. In addition to the methods that require external force field (i.e. electrical field as mentioned above, or magnetic field), strategies that rely on biochemical reaction offers intrinsically high specificity. An on-chip immunoseparation protocol ${ }^{25}$ takes advantage of specific antibodies against the translocase outer membrane protein of the mitochondria, which are immobilized on the surface of microchannels using an avidin-biotin sandwich construct. Although this affinity-based microfluidics has shown a direct retention of functional mitochondria, this method suffers from the imposed limitation that it is difficult to retrieve the isolated mitochondria.

To address the unmet need of isolating cellular organelles from small-scale samples, we present a novel and facile approach to isolate functional mitochondria from small amount of biological samples by the so-called inertial microfluidics, more specifically, by the centrifugal-based differential migration. ${ }^{26}$ Sorting particles by their inertia offers great benefits such as simple equipment and continuous batch process. Even though the number of publications concerning on-chip particles separation relying on inertia has increased exponentially over the last few years, the current applications are mostly limited to rigid particles such as polystyrene beads or cells. ${ }^{26,27}$ As a proof of concept to separate cellular organelles by centrifugal-based differential migration, we demonstrate the isolation of functional mitochondria from nuclei and cell debris with crude cell lysate of less than 100 cells.

\section{Results and discussion}

Presented here is a novel strategy to isolate cellular organelles by their intrinsic differences in inertia. Isolation of mitochondria is selected as a model organelle, for its essential and unique roles in metabolisms and cellular functions. Moreover, due to its large diversity, mitochondria present a tangible challenge when it comes to separating this organelle from other cellular components. As shown in Fig. 1, the device, hereafter

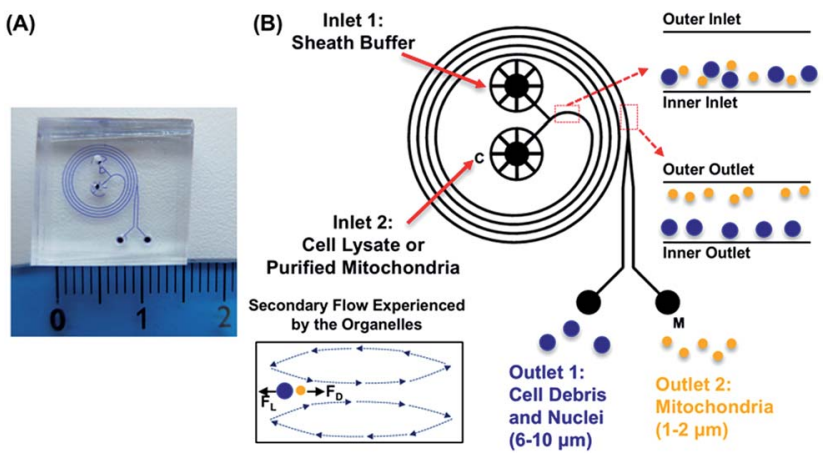

Fig. 1 Schematic representation of the mitochondria sorting by the centrifugal-based differential migration: (A) picture showing an overview of the mito chip. (B) The sheath buffer and the cell lysate/purified mitochondria are introduced into inlet 1 and inlet 2 , respectively. The organelles are separated based on the secondary flow exerted on them. Mitochondria (1-2 $\mu \mathrm{m})$, selected as the model organelle in this study, and cell debris and nuclei $(6-10 \mu \mathrm{m})$ are expected from outlet 2 (M, outer outlet) and outlet 1 (inner outlet), respectively.

referred to as the mito chip, is designed to enable effective separation between mitochondria, and nuclei/cell debris (population of 1-2 $\mu \mathrm{m}$ versus population of 6-10 $\mu \mathrm{m}$ ), while a similar design has been previously validated for separating polystyrene particles of $1.9 \mu \mathrm{m}$ and $7.32 \mu \mathrm{m} .{ }^{28}$ The inertial lift and so-called Dean drag forces collectively render particles of different sizes to migrate differently along the channel width. ${ }^{26,27}$ More specifically, mitochondria of smaller sizes compared to other cytosolic fractions (i.e. nuclei and cell debris) migrate to the outer half of the channel, whereas the larger cellular organelles move to the inner half of the channel. The size-dependent equilibrium position is determined by the inertial forces of the particulates and the Dean vortices generated by the spiral channel geometry.

Firstly, pre-isolated mitochondria from mouse liver were used to validate the capability of the device. The concentration of purified mitochondria used to feed the mito chip (see below) was predetermined using the Bradford protein assay. ${ }^{29}$ As a method to quantify the amount of retrieved functional mitochondria isolated by the mito chip, the active mitochondria were stained with a commercially available fluorescent dye, Mitotracker red. This red-fluorescent dye accumulates in mitochondria with a membrane potential - a hallmark for functional mitochondria. Both the total fluorescence intensity (ex. obtained from a fluorimeter) and the numbers of mitochondria (ex. counted by the fluorescent spots using microscopy) were measured to determine the optimal staining conditions. A linear correlation was observed between the measured signal with titrated amount of purified mitochondria (Fig. 2), which suggested that the Mitotracker staining could serve as a facile indication of the amount of retrieved active mitochondria before and after the sorting.

To investigate whether the mitochondria migrate towards the outer ring of the mito chip, purified mouse liver mitochondria (214 $\mathrm{ng} \mu \mathrm{L}^{-1}$ ) were introduced into the inlet (Fig. 1B, inlet 2, marked with a "C"), while the sheath buffer (Mito buffer) 
(A)

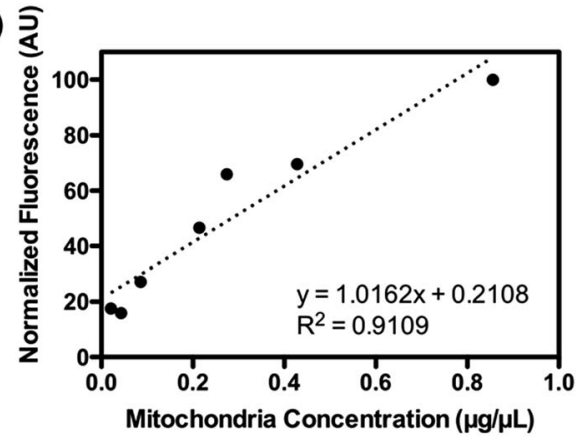

(B)

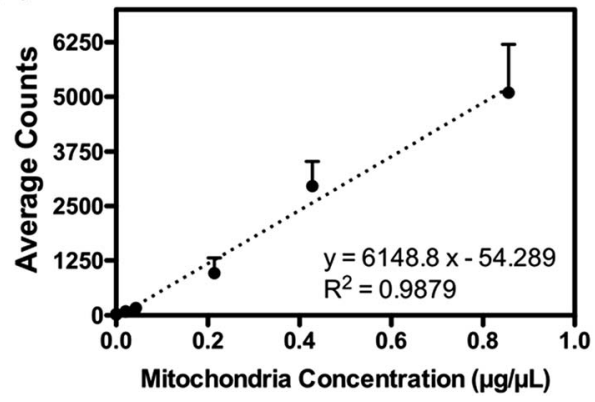

Fig. 2 Mitotracker red calibration. A fixed amount of Mitotracker red was used to stain titrated amount of mitochondria. Both the (A) measured fluorescence and (B) average counts of fluorescent spots obtained by the microscopy suggested linear correlations with the titrated amount of purified mitochondria.

was introduced into the other (Fig. 1B, inlet 1). Samples collected from the two individual outlets were analyzed subsequently without further post-processing. As observed in the representative microscopic images shown in Fig. 3, the mitochondria mostly migrated towards the intended outlet (outlet 2, marked with a "M"), whereas only traces of mitochondria were collected from the other outlet (outlet 1). Similar results were obtained by measuring the total fluorescence after Mitotracker staining (data not shown). For the ease of quantification, the subsequent quantitative analysis was conducted mainly using the total fluorescence obtained by Mitotracker staining as an indication of the retrieved amount of active mitochondria.

As mentioned previously, the isolation relied on the migration of mitochondria when experiencing the centrifugal-based differential migration. As illustrated in Fig. 1B (lower left corner), the centrifugal acceleration is determined by the magnitude of the secondary flows dominated by the so-called Dean number, which increases with the increase of fluid velocity. ${ }^{30}$ Although the stronger the Dean vortices, the stronger the centrifugal force acting on the fluid, greater recirculation of flow is counter-productive to the particle separation. ${ }^{28}$ According to previous studies, the flow is thus limited to De $<1$ (in this case, $\mathrm{De}=0.66$, Uf $=66.7 \mathrm{~mm} \mathrm{~s}^{-1}$ ). However, as the physical properties (size, density) of mitochondria remain undetermined, the optimized flow rate of sorting was determined empirically. As shown in Fig. 4, up to $90 \%$ of mitochondria were retrieved from outlet 2 , when using a flow rate of $20 \mu \mathrm{L} \mathrm{min}$. The optimized volumetric flow rate is in line with what was
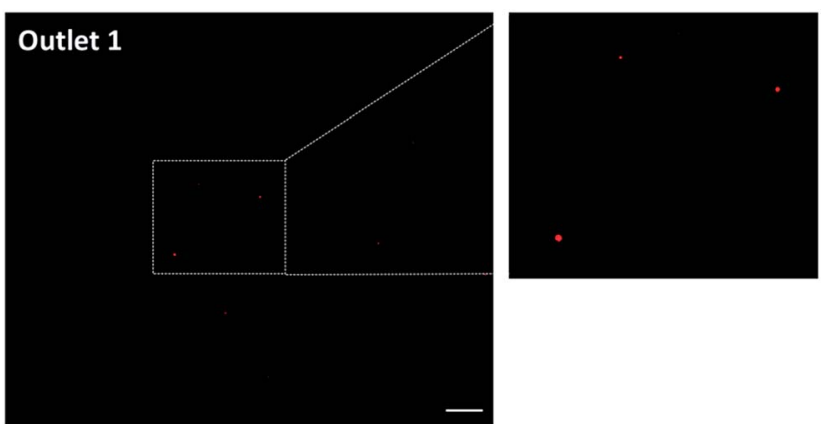

\section{Outlet 2:}

Mitochondria

Expected
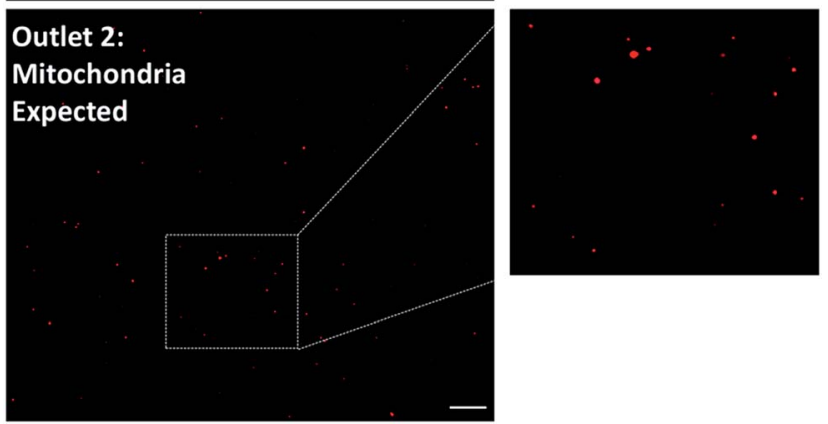

Fig. 3 Microscopic observation of the retrieved samples from the two outlets. The red fluorescent spots represent the functional mitochondria stained with Mitotracker red. Observed from the representative images, minimal mitochondria were collected from outlet 1 , whereas a multitude of mitochondria were observed from outlet 2 . Scale bar: $20 \mu \mathrm{m}$.

observed using polystyrene particles of $1.9 \mu \mathrm{m}$ and $7.32 \mu \mathrm{m} .{ }^{28}$ However, less complete separation was observed, most likely due to the heterogeneity of mitochondria compared to polystyrene particles.

To further validate that the retrieved mitochondria retain their biological functionality, the apurinic/apyrimidinic endonuclease 1 (APE1) activity of unsorted and sorted liver mitochondria was measured. APE1 is an essential DNA repair enzyme involved in the maintenance of mitochondrial DNA stability and thereby maintenance of mitochondrial function. ${ }^{31}$ The measured APE1 activity was normalized to the total amount of protein to fairly assess the repair activity per unit of mitochondria. As shown in Fig. 5, the mitochondria collected from

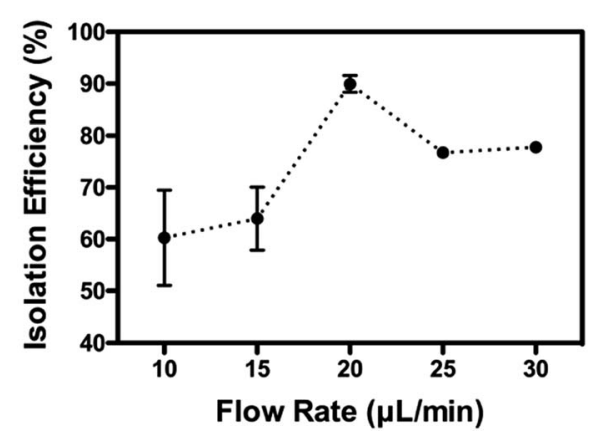

Fig. 4 Optimization of the isolation efficiency: isolation of the mitochondria by their inertial properties is optimized by operating the sorting at different flow rates. Presented results are from 3 different experiments (mean value \pm S.E.M.). 
outlet 2 exhibited a higher DNA repair activity per microgram of protein than the unsorted sample and the sample collected from outlet 1. Mitochondrial function such as DNA repair activity is thought to associate with the morphology of the mitochondria. It would be appealing to study why the mitochondria of the two populations differ with regard to their repair activities. ${ }^{32}$

A subsequent effort was to address whether the mito chip may be applied for isolation of mitochondria from a small scale biological relevant sample. For this purpose, HeLa cells were selected as a model. Crude cell lysate was prepared following standard protocols. ${ }^{13,33}$ Briefly, the cells were homogenized by lysing the cell membranes in a hypotonic buffer followed by mechanical disruption with a DOUNCE glass homogenizer. Two hundred $\mu \mathrm{L}$ of the cell lysate was then introduced into the inlet (inlet 2, marked with a "C") without further pre-processing, while the sheath buffer (Mito buffer) was introduced into the other (inlet 1). Samples from the two individual outlets were collected without further post-processing and then analyzed by Mitotracker staining as previously described. As observed in Fig. 6, a relatively high amount of active mitochondria was retrieved from the outlet 2 , to where the mitochondria were expected to migrate, whereas only minimal fraction of mitochondria was collected from outlet 1 . Although large nuclei and

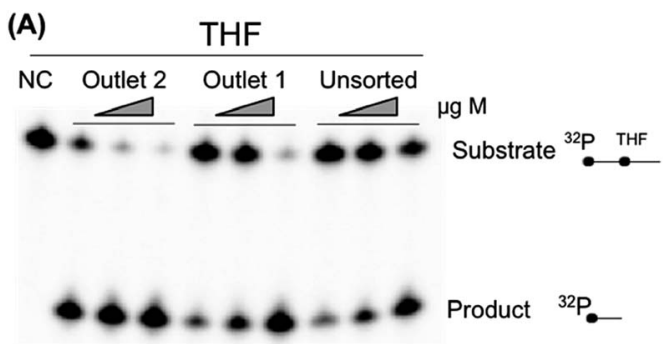

(B)

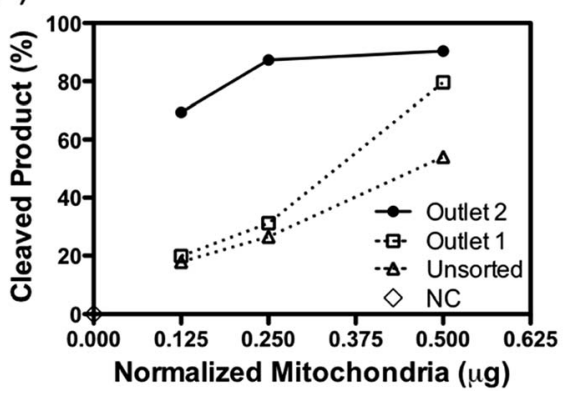

Fig. 5 DNA repair capacity assayed as the apurinic/apyrimidinic endonuclease 1 (APE 1) activity. (A) Representative gel: titrated amounts of mitochondria from the unsorted and retrieved samples from outlet 1 and outlet 2 were incubated with a THF-containing double stranded oligonucleotide, which is a specific substrate for APE1. The product band in the gel indicates the DNA repair activity in the samples. NC: negative control without mitochondria. (B) Quantification of the cleaved product: the mitochondria collected from outlet 2 exhibited a higher DNA repair activity per microgram of protein (normalized to the total amount of protein) than the unsorted sample and the sample collected from outlet 1 . Filled circle: retrieved sample from outlet 2; empty square: retrieved sample from outlet 1; empty triangle: unsorted sample from inlet 2; empty diamond: negative control without mitochondria.
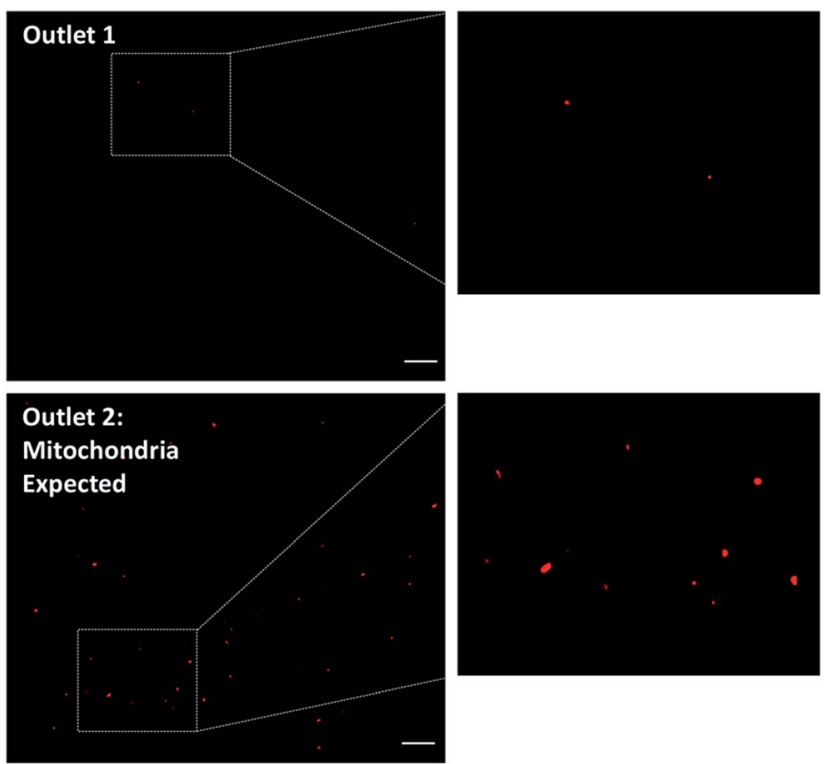

Fig. 6 Isolation of active mitochondria from crude cell lysate. $200 \mu \mathrm{L}$ of crude cell lysate from HeLa cells without pre-treatment was used. Representative microscopic observations demonstrated greater amount of active mitochondria retrieved from outlet 2 (right) as compared to outlet 1 (left). Scale bar: $20 \mu \mathrm{m}$.

cell debris were observed to non-specifically absorb onto the channel surface, rendering an altered flow profile, the isolation remained functional. The results clearly demonstrated the potential of the mito chip in isolating functional mitochondria from biological crude samples (i.e. isolation of $200 \mu \mathrm{L}$ sample in 10 minutes). Perhaps more importantly when it comes to translating the technology into clinical relevant analysis, no delicate equipment but only a standard syringe pump is required for the separation.

As above-described, non-specific absorption of cell debris was observed when crude cell lysate was used directly for the sorting. The clogging may pose a problem when a large volume of sample is intended for the sorting. To minimize the potential clogging issue under such conditions, a brief pre-centrifugation was introduced to remove large fragments of the cell debris (detailed in the Method section). The sorting was then operated as previously described and the experiments in the following have been made using HEK293T cells as an additional model. As shown in Fig. 7, a greater amount of active mitochondria was consistently retrieved from outlet 2 compared to outlet 1 . Of particular note is that the isolation functioned most effectively when optimal concentrations of cells were used (71-708 cells per $\mu \mathrm{L},>60 \%$ isolation efficiency). This observation most probably reflects that more cellular contents may affect the flow profile, rendering less optimal sorting (i.e. 3540 cells per $\mu \mathrm{L}$ ). Furthermore, the Western blotting analysis has been performed to check the purity of the isolated mitochondria. As evident from Fig. 8, a clear band corresponding to the Ku86 protein (used as the marker for the nuclei) was visualized only in the positive control (WCE: Whole Cell Extract), while the samples sorted from the outlets contain no trace (outlet 2) or very limited 


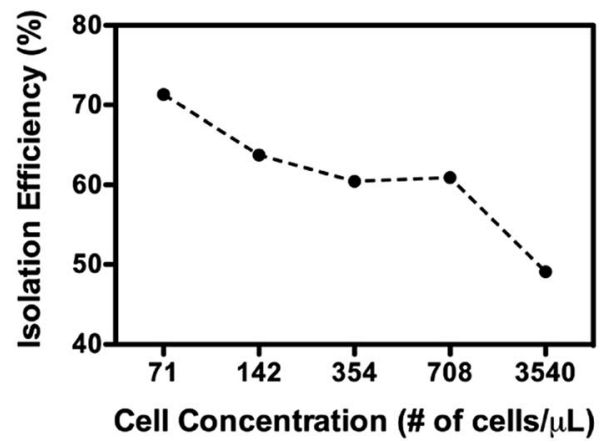

Fig. 7 Isolation of active mitochondria from crude cell lysate pretreated by brief centrifugations. Fluorescence from Mitotracker red measured the amount of active mitochondria. $X$-Axis is the concentration of cells.

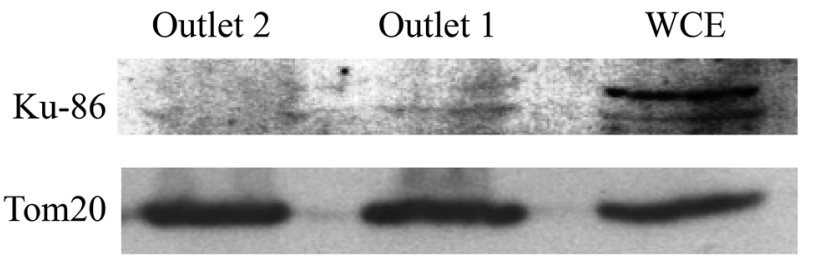

Fig. 8 Western blot analysis for the purity of isolated samples. $15 \mu \mathrm{g}$ of total proteins (quantified by Bradford) of samples isolated from outlet 1 and outlet 2 were subjected to western blot analysis using Ku86 and Tom 20 as markers for the nuclei and the mitochondria, respectively. 5 $\mu \mathrm{g}$ of total protein from HEK293T whole cell extract (WCE) were used as positive control. Representative images were obtained from 3 independent western blotting.

trace (outlet 1) of the nuclear protein. Instead, in all the 3 samples a clear band for the mitochondrial specific protein Tom 20 was witnessed. Same results were obtained using antilamin B antibody as a nuclear marker (data not shown). These observations suggested that only pure mitochondria without any nucleic contaminants were collected from the anticipated outlet, that is the outlet 2 . Last but not least, with the brief precentrifugation to precipitate the cell debris, the mito chip remained functional for processing at least up to $2 \mathrm{~mL}$ of crude cell lysate.

\section{Conclusions}

Isolation of cellular organelles from samples of clinical relevant scale has posed a significant challenge to the currently available isolation methods. To confront this issue, this study presents a novel and facile approach to isolate active mitochondria based on the centrifugal-based differential migration. Functional mitochondria have been successfully isolated from crude cell lysate even with less than 100 cells. Aside from the possibility for small-scale analysis, the presented methodology offers many distinct features, including the simple procedures, minimal equipment demands and continuous processing. With this platform capable of isolating functional mitochondria particularly from a small amount of sample, many unmet clinical possibilities are anticipated. For instance, it becomes practical to obtain mitochondria from the patient samples, and to elucidate how defective mitochondria link to the human diseases such as cancers, premature aging syndromes, diabetes and neurodegenerative disorders. Furthermore, the isolation may be tailored for an array of subcellular fractions, rendering more efficient identifications and characterizations of intracellular organelles of interest and, consequently, advancing the study of biology continuously.

\section{Experimental methods}

\section{Design and fabrication of the chip}

The chip was designed based on previous studies ${ }^{28}$ with slight modifications. The design was overviewed as a 4-loop spiral geometry with two inlets and two bifurcating outlets. The channel width was $100 \mu \mathrm{m}$ with $250 \mu \mathrm{m}$ spacing between two successive loops. The initial radius of curvature of the spiral was $1.5 \mathrm{~mm}$ and the total length of the microchannels was $\sim 9 \mathrm{~cm}$. Fabrication of the chip followed standard soft-lithography techniques, ${ }^{34}$ casting and curing the PDMS prepolymer on a SU-8 3025 (MicroChem) master of a channel height at around $50 \mu \mathrm{m}$. PDMS prepolymer (Sylgard 184) was prepared in a $10: 1$ (base : curing agent) ratio and cured at $65{ }^{\circ} \mathrm{C}$ for $1 \mathrm{~h}$. After curing, the PDMS strip was peeled off from the SU-8 master, and the input and output ports were cored using a hole puncher (Miltex Biopsy Punch with Plunger, ID $1.0 \mathrm{~mm}$, OD $1.27 \mathrm{~mm}$, Ted Pella, CA, USA). The PDMS strip was then $\mathrm{O}_{2}$ plasma treated (30 s at $20 \mathrm{~W}$, Plasma Asher, Quorum Technologies, West Sussex, RH, USA) and bonded to a $1 \mathrm{~mm}$ thick PDMS strip to completely seal the microchannel. Prior to the experiments, the channel was wetted with the Mito buffer (400 mM sucrose, $10 \mathrm{mM}$ TES, $100 \mu \mathrm{M}$ EGTA and $2 \mu \mathrm{M}$ DTT supplemented with phosphatases and proteases inhibitors) for at least $15 \mathrm{~min}$. The syringe pump (Harvard Apparatus) was used to control the flow rates of reagents.

\section{Preparation of purified mouse liver mitochondria}

Mouse liver mitochondria (from $\mathrm{C} 57 \mathrm{BL} / 6 \mathrm{~J}$ mice) were isolated as previously described..$^{35}$ Briefly, the liver was homogenized in 10 volumes $(\mathrm{w} / \mathrm{v})$ of ice-cold isolation buffer containing $210 \mathrm{mM}$ mannitol, 70 mM sucrose, 5 mM HEPES, 1 mM EDTA, pH 7.35, in the presence of $1.4 \mathrm{U}$ of subtilisin A per $\mathrm{mL}$ of buffer. The homogenate was transferred to a centrifuge tube and centrifuged for $10 \mathrm{~min}$ at $8500 \times g$. The supernatant was discarded and the pellet was resuspended in the same volume as in the homogenization step with protease inhibitors $(0.15 \mathrm{mM}$ spermine, $0.75 \mathrm{mM}$ spermidine, $1 \mathrm{mM}$ PMSF, $5 \mathrm{mM}$ DTT, 1:1000 protease inhibitor cocktail set III; Calbiochem), and centrifuged for 10 minutes at $500 \times g$ (twice). After that, the liver mitochondria were obtained after centrifugation of the supernatant at $10000 \times g$ for $10 \mathrm{~min}$. The mitochondrial pellets were resuspended in $1 \mathrm{~mL}$ of isolation buffer without EDTA. All the above procedures were performed at $4{ }^{\circ} \mathrm{C}$. Mitochondrial protein concentration was measured by the Bradford protein assay. ${ }^{29}$ 


\section{AP endonuclease assay}

APE1 incision activity in liver mitochondria was assayed using a DNA oligonucleotide containing the AP site analog tetrahydrofuran (THF) (5'-ATA TAC CGC CGX CGG CCG ATC AAG CTT ATT-3', Sigma ( $\underline{\mathrm{X}}$ indicates the position of THF)). The oligonucleotide was radioactively labeled at the $5^{\prime}$-end using $\gamma$-[32P]ATP (Perkin-Elmer) and T4 polynucleotide kinase (Thermo Scientific). Microspin columns G50 were used to remove the unincorporated ATP (Amersham Bioscience). The labeled oligonucleotide was annealed to a complementary strand (5'AAT AAG CTT GAT CGG CCG GCG GCG GTT ATA T-3') and used as a substrate.

Mitochondrial proteins were extracted from the isolated liver mitochondria by dilution of whole mitochondria (WM) in a buffer containing $20 \mathrm{mM}$ HEPES/KOH ( $\mathrm{pH}$ 7.6), 1 mM EDTA, $2 \mathrm{mM}$ DTT, $5 \%$ glycerol, and $300 \mathrm{mM} \mathrm{KCl}$ for 2 minutes followed by dilution in the same buffer excluding the $\mathrm{KCl}$ to give a final concentration of $100 \mathrm{mM} \mathrm{KCl}$. APE1 activity was measured by incubation of $0.125,0.25$ and, $0.5 \mu \mathrm{g}$ purified liver mitochondria with 15 nmoles of $32 \mathrm{P}$ end-labeled DNA substrate containing the THF for 30 minutes at $37^{\circ} \mathrm{C}$, in a $20 \mu \mathrm{L}$ reaction volume containing $20 \mathrm{mM}$ HEPES $\mathrm{pH}$ 7.6, $5 \mathrm{mM}$ EDTA, $75 \mathrm{mM} \mathrm{KCl}$, $1 \mathrm{mM} \mathrm{MgCl}, 2$, 5\% glycerol, and $5 \mathrm{mM}$ DTT. Reactions were terminated by addition of $0.4 \%$ SDS and $0.2 \mu \mathrm{g} \mu \mathrm{L}^{-1}$ of proteinase $\mathrm{K}$, and by 15 minutes incubation at $55^{\circ} \mathrm{C}$. Finally, the samples were mixed with $20 \mu \mathrm{L}$ of formamide dye and heated for 2 minutes at $90{ }^{\circ} \mathrm{C}$. Substrates and products were separated using a $20 \%$ denaturing polyacrylamide gel, and the radioactively labeled DNA was visualized using storage phosphor screens (Amersham Bioscience), scanned using a Personal Molecular Imager ${ }^{\mathrm{TM}}$ and quantified using Image Lab software (Bio-Rad).

\section{Cell culture and lysate preparation}

All medium, medium components and reagents were from Sigma Aldrich. Human HEK293T and HeLa cells (purchased from Leibniz Institute DSMZ-German Collection of Microorganisms and Cell Cultures, Braunschweig, Germany) were grown in DMEM supplemented with $10 \%$ fetal bovine serum, $2 \mathrm{mM}$ L-glutamine and $1000 \mathrm{U} \mathrm{mL}^{-1}$ penicillin-streptomycin. To prepare cell lysate cells were trypsinized and resuspended in $50 \mu \mathrm{L}$ (per million of cells) of pre-chilled HIM buffer $(15 \mathrm{mM}$ $\mathrm{MgCl}_{2}, 10 \mathrm{mM}$ tris-[3-( $N$-morpholino)propanesulfonic acid] $\mathrm{pH}$ 7.4, $10 \mathrm{mM} \mathrm{KCl}$ ) supplemented with phosphatases and proteases inhibitors. After 10 minutes of incubation at $4{ }^{\circ} \mathrm{C}$ in $50 \mu \mathrm{L}$ (per million of cells) of pre-chilled Mito buffer (400 mM sucrose, $10 \mathrm{mM} \quad \mathrm{N}$-tris(hydroxymethyl)methyl-2-aminoethanesulfonic acid, $100 \mu \mathrm{M}$ ethylene glycol tetraacetic acid and $2 \mu \mathrm{M}$ dithiothreitol pH 7.2 supplemented with phosphatases and proteases inhibitors) were added and the cells were homogenized by using a tissue grinder dounce 0.5 (\#885300, Kimble Chase). An average of 50 strokes were made and cell breakage was checked at the microscope using trypan blue. Where reported the cell lysate was centrifuged 10 minutes at $900 \mathrm{~g}$ and the supernatant collected in an ice-cold tube prior to insertion in the microchip inlet.

\section{Quantification of functional mitochondria}

The collected sample from both outlets, as well as the sample introduced into the outlet were stained with $2.5 \mu \mathrm{M}$ Mitotracker red (Molecular Probes Inc., Eugene, OR) at $25{ }^{\circ} \mathrm{C}$ for $5 \mathrm{~min}$. The amount of mitochondria was determined by two different approaches. To verify whether there were nuclei in the retrieved sample, $5 \mu \mathrm{L}$ of sample was mixed with $5 \mu \mathrm{L}$ of ProLong (containing DAPI) and placed onto a slide glass, covered by a cover glass. To quantify the amount of mitochondria, total fluorescence was measured directly using a qPCR machine using the mode for the endpoint fluorescence (Agilent Mx3000P). The isolation percentage of functional mitochondria was defined as: fluorescence $_{\text {outlet2 } 2} /$ fluorescence $_{\text {outlet1 }}+$ fluorescence $_{\text {outlet2 }}$ ).

\section{Microscopy and image analysis}

Slides were analyzed with Olympus IX73 epifluorescence microscope with an oil objective (Uplansapo $60 \times, \mathrm{NA}=1.35$, Olympus). The fluorophores were excited by a mercury lamp. Fluorescent signals from Mitotracker red were selectively filtered through a dichroic (U-FGNA FL filter cube, DM570) and bandpass filters (excitation: BP 540-550, emission: BP 575-625) before being collected by an Andor Zyla sCMOS camera. Image acquisition was operated by the software of CellSense Dimension (Olympus). At least five images were recorded, for each sorting condition unless otherwise cited. ImageJ was then used to analyze and quantify the number of fluorescence spots (size: 0-infinity pixel, ${ }^{2}$ circularity: 0-1).

\section{Western blot analysis}

Samples sorted from outlet 1 and outlet 2 were centrifuged 10 minutes at $10000 \mathrm{~g}$ at $4{ }^{\circ} \mathrm{C}$. The pellets obtained were lysed using a buffer containing $10 \mathrm{mM}$ Tris- $\mathrm{HCl}(\mathrm{pH} 7.5), 5 \mathrm{mM}$ EDTA, $2 \mathrm{mM}$ PMSF and $0.25 \mathrm{mg} \mathrm{mL}^{-1} \beta$-glycerophosphate followed by incubation on ice for 10 minutes. As positive control a whole cell extract from $10^{6}$ HEK293T cells was obtained using the same procedure. Protein concentration was determined by the Bradford protein assay (Sigma). Samples were then mixed with SDS loading buffer ( $2 \%$ SDS, $2 \mathrm{mM} \beta$-mercapto-ethanol, $4 \%$ (v/v) glycerol, $50 \mathrm{mM}$ Tris- $\mathrm{HCl} \mathrm{pH} \mathrm{7,} \mathrm{0.05 \%} \mathrm{bromophenol}$ blue) and loaded on a $10 \%$ SDS gel. PageRuler prestained Protein ladder from Thermo Scientific was used as a size marker. The gel was run at $200 \mathrm{~V}$ for 30 minutes in $1 \times$ SDS running buffer (25 mM Tris-HCl, $192 \mathrm{mM}$ glycine, 0.1\% SDS). The separated proteins were blotted onto a $0.45 \mu \mathrm{m}$ nitrocellulose membrane at 30 volts for 18 hours in transfer buffer (25 mM Tris- $\mathrm{HCl}, 192 \mathrm{mM}$ glycine, $0.1 \%$ SDS, 20\% ethanol) at $4{ }^{\circ} \mathrm{C}$. The nitrocellulose membrane was incubated in blocking buffer (10 mM Tris pH 7.5, $500 \mathrm{mM} \mathrm{NaCl,} \mathrm{0.25 \%} \mathrm{Tween} 20$ and $5 \%$ skimmed milk powder). Subsequently the membrane was incubated over night at $4{ }^{\circ} \mathrm{C}$ with either Ku 86 or Tom 20 primary antibodies (Santa Cruz) diluted in blocking buffer. The membrane was washed $3 \times 30$ minutes in TBS buffer $(20 \mathrm{mM}$ Tris $\mathrm{pH} 7.5,0.5 \mathrm{M} \mathrm{NaCl}, 0.25 \%$ Tween 20 ) at room temperature and probed with horseradish peroxidase-conjugated secondary antibodies (Dako) diluted in blocking buffer. The membrane 
was washed in TBS buffer at room temperature and bound antibodies were detected by autoradiography using the ECL Plus Western Blotting Detection Reagents (GE healthcare). Blots were exposed to hyperfilm (Kodak) for appropriate time periods in order to obtain a clear visualization of the blotted bands into the membrane.

\section{Acknowledgements}

The authors would like to acknowledge the support from the Start-Up fund and Direct Research Grant provided by the Chinese University of Hong Kong, the Lundbeck Foundation (R95-A10275), Velux Foundation, The Danish Research Council, Karen Elise Jensen Foundation, the Dagmar Marshalls Fond, the Aase and Ejnar Danielsens Foundation and the Arvid Nilssons Fond. C. T. would like to acknowledge the support of Postdoc Fellowship from the Carlsberg foundation.

\section{References}

1 X. Liu, C. N. Kim, J. Yang, R. Jemmerson and X. Wang, Cell, 1996, 86, 147-157.

2 J. Yang, Science, 1997, 275, 1129-1132.

3 S. J. Riedl and G. S. Salvesen, Nat. Rev. Mol. Cell Biol., 2007, 8, 405-413.

4 W. X. Zong, J. D. Rabinowitz and E. White, Mol. Cell, 2016, 61, 667-676.

5 K. Księzakowska-Łakoma, M. Zyła and J. R. Wilczyński, Przegl. Menopauzalny, 2014, 18, 136-144.

6 E. Gottlieb and I. P. M. Tomlinson, Nat. Rev. Cancer, 2005, 5, 857-866.

7 M. Scheibye-Knudsen, D. L. Croteau and V. A. Bohr, Mech. Ageing Dev., 2013, 134, 275-283.

8 P.-M. Badin, et al., Sci. Rep., 2016, 6, 26442.

9 T. Brun and P. Maechler, Biochim. Biophys. Acta, Mol. Cell Res., 2016, 1863, 2540-2549.

10 M. Flint Beal, Mitochondrial Biol. New Perspect., 2008, pp. 183-192.

11 R. N. Lightowlers, R. W. Taylor and D. M. Turnbull, Science, 2015, 349, 1494-1499.

12 E. Fernández-Vizarra, G. Ferrín, A. Pérez-Martos, P. Fernández-Silva, M. Zeviani and J. A. Enríquez, Mitochondrion, 2010, 10, 253-262.
13 C. Frezza, S. Cipolat and L. Scorrano, Nat. Protoc., 2007, 2, 287-295.

14 M. Toner and D. Irimia, Annu. Rev. Biomed. Eng., 2005, 7, 77103.

15 D. Mark, S. Haeberle, G. Roth, F. von Stetten and R. Zengerle, Chem. Soc. Rev., 2010, 39, 1153-1182.

16 J. Liu, Y. Lan, Z. Yu, C. S. Y. Tan, R. M. Parker, C. Abell and O. A. Scherman, Acc. Chem. Res., 2017, 50(2), 208-217.

17 T. Schneider, J. Kreutz and D. T. Chiu, Anal. Chem., 2013, 85, 3476-3482.

18 E. K. Sackmann, A. L. Fulton and D. J. Beebe, Nature, 2014, 507, 181-189.

19 H. Lu, S. Gaudet, M. a. Schmidt and K. F. Jensen, Anal. Chem., 2004, 76, 5705-5712.

20 P. Lundberg and I. Ericson, Biochem. Biophys. Res. Commun., 1975, 65, 530-536.

21 J. Sánchez-Prieto and M. J. López-Pérez, Biochim. Biophys. Acta, Biomembr., 1984, 778, 81-86.

22 D. T. Plummer, Biochem. J., 1965, 96, 729-732.

23 I. Ericson, Biochim. Biophys. Acta, Biomembr., 1974, 356, 100107.

24 G. G. Wolken, V. Kostal and E. A. Arriaga, Anal. Chem., 2011, 83, 612-618.

25 S. Kayo, J. Bahnemann, M. Klauser, R. Pörtner and A.-P. Zeng, Lab Chip, 2013, 13, 4467-4475.

26 J. Zhang, S. Yan, D. Yuan, G. Alici, N.-T. Nguyen, M. Ebrahimi Warkiani and W. Li, Lab Chip, 2016, 16, 10-34.

27 D. Di Carlo, Lab Chip, 2009, 9, 3038-3046.

28 A. A. S. Bhagat, S. S. Kuntaegowdanahalli and I. Papautsky, Lab Chip, 2008, 8, 1906-1914.

29 M. M. Bradford, Anal. Biochem., 1976, 72, 248-254.

30 W. R. Dean, London, Edinburgh, Dublin Philos. Mag. J. Sci., 1927, vol. 4, pp. 208-223.

31 M. D. Errico, E. Parlanti, E. Dogliotti, M. D'Errico and M. D'Errico, Mutat. Res., Rev. Mutat. Res., 2008, 659, 4-14.

32 D. C. Chan, Annu. Rev. Genet., 2011, 46, 265-287.

33 G. Filomeni, S. Piccirillo, I. Graziani, S. Cardaci, A. M. Da Costa Ferreira, G. Rotilio and M. R. Ciriolo, Carcinogenesis, 2009, 30, 1115-1124.

34 D. Qin, Y. Xia and G. M. Whitesides, Nat. Protoc., 2010, 5, 491-502.

35 R. Gredilla and T. Stevnsner, in DNA Repair Protocols, ed. L. Bjergbæk, Humana Press, Totowa, NJ, 2012, pp. 289-304. 